\title{
Inter-relações entre os índices financeiros setoriais da Bolsa de Valores de São Paulo e o índice Ibovespa
}

\author{
Edson Zambon Monte ${ }^{1}$
}

\begin{abstract}
Resumo: Esta pesquisa verificou as inter-relações entre os índices setoriais da BOVESPA e o índice global IBOVESPA. Adotou-se o modelo vetorial autorregressivo (VAR) e o teste de cointegração de Johansen. Os resultados demonstraram que não existe relação de longo prazo (cointegração) entre os índices. Entretanto, existe uma interdependência entre os índices no curto prazo, revelando que os efeitos positivos ou negativos podem ser disseminados entre os índices analisados, que podem, por sua vez, impactar diretamente nas decisões de investimentos dos agentes econômicos e financeiros, especialmente no que tange à diversificação de suas carteiras de ativos.
\end{abstract}

Palavras-chave: IBOVESPA. Índices Financeiros Setoriais. Vetor Autorregressivo.

Classificação JEL: G17, C32, C58.

\section{Interrelationships between the stock market sector indexes of the São Paulo Stock Exchange and the Ibovespa index}

\begin{abstract}
This paper aims to verify the interrelationships between the stock market sector indexes of the São Paulo Stock Exchange and the IBOVESPA index. The autoregressive vector model and the Johansen cointegration test were used. Results showed there is no long-term relationship (cointegration) between the indexes. However, there is an interdependence between the indexes in the short term, revealing that the positive or negative effects can be disseminated among the indices analyzed, which can directly impact the investment decisions of economic and financial agents, especially regarding the diversification of their asset portfolios.
\end{abstract}

Keywords: IBOVESPA. Stock Market Sector Indexes. Autoregressive Vector.

1 Doutor. Professor do Departamento de Economia e membro do Grupo de Pesquisa em Econometria (GPE) da Universidade Federal do Espírito Santo (UFES). E-mail: edsonzambon@yahoo.com.br 


\section{Introdução}

Embora seja de amplo conhecimento que o mercado de capitais tem um importante papel no desenvolvimento de qualquer economia, especificamente no Brasil, os benefícios advindos desse mercado foram tardios, uma vez que o país viveu um longo período de alta taxa de inflação e de instabilidade econômica, que geraram efeitos negativos sobre o mercado de ações. O Plano Real foi um marco para o mercado de ações do país e iniciou um vigoroso padrão de crescimento, especialmente a partir do ano de 2003 (PADMANABHAN, 2015). De acordo com Nunes, Costa e Meurerii (2005), em função desse desenvolvimento, a capitalização por meio do mercado de ações cresceu tanto em termos de volume de negócios, quanto em níveis de eficiência alocativa. Ainda segundo os autores, o mercado de ações do Brasil tem despertado a atenção de investidores e empresas, uma vez que o mesmo tem se mostrado como uma oportunidade para investidores externos que objetivam a diversificação de seus portfólios. Cabe mencionar que esse comportamento também pode ser observado em termos dos investidores internos.

Ressalta-se que o mercado de ações do Brasil é fortemente concentrado em um número reduzido de empresas, diferentemente da maioria dos países desenvolvidos. Os setores mais representativos do Índice da Bolsa de Valores de São Paulo (IBOVESPA), considerando a carteira do dia 05/09/2017 foram o bancário; de extração de petróleo e gás; de mineração de metais; de geração, transmissão e distribuição de energia elétrica e da indústria de bebidas. Logo, qualquer efeito negativo nas empresas que compõem esses setores pode ocasionar significativos reflexos negativos no IBOVESPA e na economia. Conforme Hermann e Martins (2012), o crescimento do mercado de ações é, em geral, um indicador positivo para a economia, criando expectativas favoráveis, tanto para as empresas emissoras quanto para os adquirentes das ações. No entanto, mesmo com relevantes externalidades positivas, o mercado de ações não está isento de riscos, assim como qualquer outro segmento do mercado financeiro. Ainda segundo Hermann e Martins (2012), perdas de recursos por parte dos investidores individuais e instituições financeiras podem ocorrer e, dependendo da proporção das perdas, desencadear uma série de efeitos de contágio, isto é, impactos sobre outros investidores e instituições, segmentos do mercado financeiro e até mesmo sobre o setor não financeiro.

De acordo com Jubert et al. (2008), os investidores globais, no intuito de diversificarem seus investimentos, focam suas atenções na dinâmica dos mercados internacionais. No caso dos investidores focados no mercado brasileiro, a abordagem setorial é primordial no que se refere à redução do risco de carteiras de ações. Conforme os autores, é de se esperar que as ações de empresas que atuam em uma mesma atividade econômica apresentem comportamentos similares no mercado de ações. Isso decorre do fato de que as empresas de um mesmo setor são, em geral, afetadas pelos mesmos fatores do ambiente competitivo e são norteadas pela mesma 
legislação. Logo, para o investidor dedicado a um determinado mercado, torna-se essencial a diversificação setorial para reduzir o risco não sistemático. Cabe mencionar que, para Baca, Garbe e Weiss (2000), em estudo sobre os sete principais mercados de ativos do mundo, a influência dos componentes específicos de cada país sob a variação dos retornos das ações vem declinando, ao passo que o impacto dos componentes setoriais tem permanecido relativamente constante ou tem aumentado.

No Brasil, poucos são os estudos que abordam os índices financeiros setoriais, especialmente no que tange às pesquisas econométricas. Entre eles, pode-se citar Jubert et al. (2008), que analisaram o padrão da volatilidade dos índices de ações do mercado brasileiro, no período de 2006 a 2007, a saber: IBOVESPA e os índices setoriais de Energia Elétrica (IEE), de Sustentabilidade Empresarial (ISE), do Setor Industrial (INDX) e de Telecomunicações (ITEL). Os autores utilizaram modelos de heteroscedasticidade condicional da família GARCH. Medeiros (2012) utilizou essa mesma abordagem para analisar a volatilidade de quatro índices financeiros setoriais da Bovespa e Ferreira e Mattos (2012) analisaram o efeito contágio da crise financeira internacional do subprime sobre o índice IBOVESPA e sobre alguns índices de mercado setoriais, a partir do estudo do padrão das covariâncias estimadas entre os índices do mercado acionário brasileiro e americano. A análise empírica foi baseada nos modelos multivariados GARCH-BEKK.

No entanto, não existem estudos que verifiquem as inter-relações entre os índices setoriais da Bolsa de Valores de São Paulo e o índice IBOVESPA em termos de correlação temporal. Dessa forma, o objetivo desta pesquisa foi analisar as interrelações entre o índice global IBOVESPA e alguns dos índices setoriais da Bovespa, a saber: Índice de Consumo (ICON); IEE; Índice Financeiro (IFNC); INDX; e, Índice de Materiais Básicos (IMAT).

Destaca-se que as análises sobre as relações de séries econômicas e financeiras têm avançado nos últimos anos, adotando metodologias como o método wavelets (método de análise de ondaletas) e os métodos não paramétricos de causalidade de Granger. Reboredo, Rivera-Castro e Ugolini (2017), por exemplo, usaram wavelets e testes lineares e não lineares de causalidade de Granger, a fim de examinar a dependência e a direção da causalidade entre os retornos de reservas de petróleo e de energia renovável em diferentes escalas temporais. Especificamente em relação às ondaletas (método wavelets), essas são funções que possibilitam o fracionamento das séries temporais em duas subséries (de altas e de baixas frequências), o que permite um melhor estudo do comportamento das mesmas (GENÇAY; SELÇUK; WHITCHER, 2002). Porém, este artigo baseou-se em metodologias tradicionais para estimar seus resultados, tais como: teste linear de causalidade de Granger, modelo vetorial autorregressivo (VAR) e o teste de cointegração de Johansen. Para futuros trabalhos, pretende-se utilizar o método de wavelets (método de análise de ondaletas) e os métodos não paramétricos de causalidade de Granger, buscando, inclusive, fazer um paralelo com os resultados encontrados nesta pesquisa. 
O presente artigo está estruturado da seguinte forma: além desta introdução, a seção 2 traz uma revisão de literatura; na seção 3 apresentam-se o modelo vetor autorregressivo (VAR) e o teste de cointegração de Johansen; os resultados e discussões são demonstrados na seção 4; e, por último, as considerações finais são apresentadas na seção 5 .

\section{Revisão de literatura}

A questão relativa à seleção de carteiras de ativos é clássica na literatura de finanças e vem sendo estudada desde o trabalho de Markowitz (1952). Trata-se de um problema de otimização quadrática, envolvendo a maximização do retorno da carteira e a minimização da variância dado um nível de retorno (após a publicação do artigo de Markowitz, a variância passou a definir risco ${ }^{2}$ ). Markowitz (1952) foi um dos pioneiros no desenvolvimento da teoria moderna da carteira. Tal teoria descreve como os investidores utilizam o princípio da diversificação, com o objetivo de melhorar a relação risco e retorno de suas carteiras de investimentos, sendo que há um trade-off nessa relação. Para Markowitz (1952), a diversificação permite a redução do risco associado. De acordo Müller (1989), enquanto na ciência atuarial a lei dos grandes números desempenha um papel central, esse não é o caso na teoria da carteira. Devido à correlação entre os retornos sobre os ativos financeiros, a diversificação permite, em geral, apenas uma redução, mas não uma eliminação do risco. Markowitz (1952) foi o primeiro que levou em consideração as covariâncias entre as taxas de retorno. Caso haja uma forte correlação positiva entre os retornos dos ativos, tais ativos se comportariam como um ativo único, e os benefícios da diversificação seriam mitigados.

Ressalta-se que, Markowitz (1952), tomando como base algumas suposições, considera que, entre as várias combinações plausíveis, existe um conjunto de carteiras que é eficiente. De acordo com a sua teoria, o investidor deveria estar preocupado com o risco total da carteira de ativos. Nesse caso, a combinação dos ativos com risco geraria uma curva, na qual poderia ser detectada uma fronteira eficiente. Essa fronteira eficiente seria o conjunto de carteiras com a melhor relação risco e retorno (MACEDO JUNIOR, 2003). De acordo com Fuinhas, Marques e Nogueira (2014), são carteiras eficientes ${ }^{3}$ aquelas que apresentam a máxima rentabilidade

2 Vale destacar que em economia e finanças, os conceitos de risco e incerteza são diferentes: risco é mensurável, incerteza, não.

3 Ressalta-se que, segundo Müller (1989), a abordagem de Markowitz é um método para calcular carteiras eficientes de média variância. Logo, tal abordagem baseia-se na análise de média-variância, na qual a variância da taxa global de rentabilidade é tomada como uma medida de risco, e o valor esperado mede a lucratividade. Diferentemente da maximização da utilidade esperada, a análise da média-variância toma como base apenas os dois primeiros momentos da variável e não há fundamentação teórica clara. 
dados os vários níveis de risco e o risco mínimo para os vários níveis de rentabilidade. Ainda segundo os autores, a escolha da carteira ótima dependerá do investidor, considerando as suas preferências individuais em relação à rentabilidade e ao risco, refletidas nas curvas de indiferença. Segundo Markowitz (1991, p. 470), "um investidor racional, ao decidir sobre a melhor alternativa de investimento, atua levando em consideração as distribuições de probabilidade dos retornos".

Avançando em relação ao modelo de Markowitz (1952), Tobin (1958) desenvolveu a teoria da separação. Segundo o teorema da separação, a escolha da carteira ótima independe da propensão do investidor ao risco. Além disso, o teorema da separação de Tobin (1958) afirma que as decisões sobre a escolha da carteira são realizadas em duas etapas: na primeira, encontra-se a carteira eficiente de ativos arriscados; na segunda, deve-se estabelecer a fração ótima para investir na carteira eficiente de ativos livres de riscos e em ativos com risco (FUINHAS; MARQUES; NOGUEIRA, 2014). Ainda segundo Tobin (1958), em equilíbrio, o retorno de um investimento em certo ativo deve gerar dois prêmios: o do tempo e o do risco corrido. 0 primeiro refere-se à uma recompensa pelo não usufruto imediato da renda. 0 segundo, diz respeito à diferença entre os retornos pagos e a taxa livre de risco. Logo, os investidores somente aceitarão comprar um ativo com risco se o retorno esperado compensar adequadamente o risco corrido (MACEDO JUNIOR, 2003).

Do mesmo modo, com o intuito de melhorar o modelo de Markowitz (1952), Sharpe (1963) descreveu que a relação entre os ativos se deve à ligação comum com a carteira de mercado, sendo que o relacionamento entre cada título e o mercado é linear. Além disso, Sharpe (1963) elaborou um modelo de mercado no qual a variabilidade total de uma ação pode ser dividida em duas partes: i) uma parte decorre da influência do mercado, denominada de risco sistêmico ou de mercado; e ii) a segunda parte ocorre em função das características específicas da ação, relacionada às suas variações, e é chamada de risco não sistêmico ou específico de uma empresa ou ativo. Conforme Fuinhas, Marques e Nogueira (2014), o risco específico pode ser dividido em risco da ação especificamente e em risco devido às características do setor no qual a empresa está inserida. 0 risco não sistêmico pode ser eliminado ou reduzido por meio da diversificação, o que não ocorre para o risco de mercado.

Ademais, Sharpe (1964) desenvolveu a chamada teoria do mercado de capitais, que aborda o apreçamento dos ativos. 0 autor toma como base o comportamento conjunto dos indivíduos, que é uma limitação do modelo inicial de Markowitz (1952), o qual considera as decisões de investimento de forma individual. Conforme Teixeira (2011), Markowitz (1952) tinha como objetivo principal reduzir o risco não sistemático, isto é, aquele que impacta, em grande parte, as empresas e são específicos a ela. Nesse sentido, a diversificação dos ativos aparece como relevante, pois o investidor não deve alocar o capital em apenas um tipo de investimento. No que se refere à teoria de Sharpe (1964), a mesma é focada em minimizar o risco de mercado, 
ou seja, o risco que impacta todas as empresas, em maior ou menor grau (TEIXEIRA, 2011).

Ainda, de acordo com Fuinhas, Marques e Nogueira (2014), por meio das teorias descritas anteriormente, foram desenvolvidos os modelos de equilíbrio dos ativos financeiros, que determinam uma relação entre a rentabilidade e o risco esperado, definindo, assim, o risco de equilíbrio. Nesse caso, considerando a ocorrência de alterações, tanto na rentabilidade como no risco, verifica-se o ajustamento instantâneo dos preços. Dessa maneira, se na formação de uma carteira com vários títulos é verificada uma alteração na rentabilidade ou no risco, o investidor reformulará a sua carteira, comprando ou vendendo títulos, para atingir novamente a rentabilidade e o risco adequados, de acordo com seus objetivos (FUINHAS; MARQUES; NOGUEIRA, 2014).

Tomando como base as contribuições de Sharpe (1964), Lintner (1965) e Mossin (1966), desenvolveu-se o Capital Asset Pricing Model (CAPM), que estabelece uma relação entre a rentabilidade esperada e o nível de risco sistemático. No CAPM, o retorno esperado de um título está positivo e é linearmente relacionado à covariância do seu retorno com o retorno de uma carteira de mercado (TEIXEIRA, 2011). Segundo Fuinhas, Marques e Nogueira (2014), o CAPM determina os preços dos valores mobiliários, que permitem que a oferta e a procura se equilibrem e, consequentemente, produzam o equilíbrio geral do mercado. 0 modelo tem se tornado essencial na teoria moderna da carteira. Além disso, o CAPM pode ser visto sob duas óticas: i) a primeira como uma reformulação das condições de otimização necessárias para o problema da média-variância de Markowitz o qual depende, portanto, da definição de risco como variância; e ii) a segunda, é que se trata de um modelo linear de fator único, combinando os retornos esperados de um ativo a um portfólio de mercado e que serve como uma medida de ativos com risco nãodiversificável (sistemático) (FUINHAS; MARQUES; NOGUEIRA, 2014). O CAPM, proposto originalmente por Sharpe (1964), pode ser visto como um modelo de precificação de ativos com risco em equilíbrio.

Macedo Junior (2003) descreve detalhadamente estudos que apresentaram críticas à teoria moderna da carteira. Tais críticas se iniciaram a partir da verificação, por meio de estudos empíricos, de que os preços de alguns ativos poderiam apresentar tendências em certos momentos, indo contra a hipótese de eficiência dos mercados. Conforme mencionado por Silva, Barbedo e Araújo, (2015), para alguns autores, os preços de mercado seguem determinados padrões que encontram explicação na teoria de finanças comportamentais, mas não na teoria moderna da carteira. De acordo com Silva, Barbedo e Araújo (2015), teorias de finanças comportamentais começaram a se destacar quando os trabalhos de Daniel Kahneman e Vernon Smith ganharam o prêmio Nobel de Economia, em 2002. Dado que os modelos de finanças comportamentais consideram aspectos psicológicos dos seres humanos, eles permitem a possibilidade de 
irracionalidade dos agentes, buscando sanar as lacunas teóricas da teoria moderna da carteira.

\section{Vetor autorregressivo (VAR) e teste de cointegração de Johansen ${ }^{4}$}

Pode-se expressar um modelo VAR ${ }^{5}$ de ordem $p$ em função de um vetor com $n$ variáveis endógenas, $\mathrm{X}_{\mathrm{t}}$, sendo que essas se conectam por meio de uma matriz $A$, da seguinte forma:

$$
A X_{t}=B_{0}+\sum_{i=1}^{p} B_{i} X_{t-i}+B \varepsilon_{t}
$$

em que: $A$ é uma matriz $n \times n$, que define as restrições contemporâneas entre as variáveis que constituem o vetor $\mathrm{n} \times 1, X_{t} ; B_{0}$, vetor de constantes $n \times 1$; $B_{i}$, matrizes $\mathrm{n} \times \mathrm{n} ; B$, matriz diagonal $n \times n$ de desvio-padrão; e $\varepsilon_{t}$, vetor $n \times 1$ de perturbações aleatórias não correlacionadas entre si contemporânea ou temporalmente, isto é:

$$
\varepsilon_{t} \sim \text { i.i.d }\left(0 ; I_{n}\right)
$$

em que 0 é o vetor nulo; e $I$, a matriz identidade.

A Equação (1) expressa às relações entre as variáveis endógenas, geralmente advindas de um modelo econômico teoricamente estruturado, e é denominada de forma estrutural. No entanto, devido à endogeneidade das variáveis do VAR, o modelo é normalmente estimado em sua forma reduzida, dada por:

$$
X_{t}=A^{-1} B_{0}+\sum_{i=1}^{p} A^{-1} B_{i} X_{t-i}+A^{-1} B \varepsilon_{t}=\Phi_{0}+\sum_{i=1}^{p} \Phi_{i} X_{t-i}+e_{t},
$$

em que: $\Phi_{i}=A^{-1} B_{i}, i=0,1,2, \ldots, p ; \mathrm{e}, B \varepsilon_{t}=A e_{t}$.

A metodologia VAR pode ser estimada por meio do método de Mínimos Quadrados Ordinários (MQO), levando em conta principalmente a interação entre as variáveis do sistema considerado. Entre as suas principais vantagens na análise econométrica estão a obtenção das funções de impulso-resposta (FRI) e a decomposição da variância (DV).

Vale lembrar que caso as variáveis estudadas sejam cointegradas, o modelo original pode ser descrito na forma de um vetor autorregressivo de correção de erros (VECM). Engle e Granger (1987) apresentaram a cointegração da seguinte forma: os

4 A metodologia descrita aqui nesta seção está baseada em Bueno (2011) e Lütkepoh (2007).

5 Metodologia desenvolvida inicialmente por Sims (1980). 
componentes de um vetor $X_{t}(n \times 1)$ são ditos serem cointegrados de ordem $(d, b)$, denotados por $X_{t} \sim C I(d, b)$, se: i) todos os componentes de $X_{t}$ são $I(d)$; e ii) existe pelo menos um vetor não nulo $\beta$, tal que $u_{t}=X_{t}^{\prime} \beta \sim(d-b), b>0$. 0 vetor $\beta$ é chamado de vetor de cointegração.

Já a definição de cointegração de Campbell e Perron (1991) é mais abrangente, não impondo a restrição de mesma ordem de integração para a existência de cointegração. Segundo esses autores, basta que ocorra a segunda condição descrita anteriormente para que os elementos de um vetor $X_{t}$ sejam cointegrados. Nesse caso, para que haja cointegração, deverá haver pelo menos duas variáveis integradas de mesma ordem na ordem máxima de integração entre todas as variáveis. Ainda, Hansen e Juselius (1995) descrevem que uma condição necessária para encontrar uma relação de cointegração entre variáveis não estacionárias exige que somente duas das variáveis do modelo sejam integradas de ordem um I(1).

A forma mais geral de um VECM é dada por:

$$
\Delta X_{t}=\Phi X_{t-1}+\sum_{i=1}^{p-1} \Lambda_{i} \Delta X_{t-i}+e_{t}
$$

em que: $X_{t}$ é um vetor $n \times 1$ de variáveis endógenas; e $\Lambda_{i}=-\sum_{j=1+i}^{p} \Phi_{j ;} i=$ $1,2, \ldots, p-1$.

Havendo raiz unitária, significa que $\Phi(I)=0$, de modo que $\Phi=\alpha \beta^{\prime}$. Nesse caso, $\beta$ é a matriz que tem $r$ vetores de cointegração e $\alpha$ é a matriz de ajustamento, com $r$ vetores de ajustamento. 0 modelo de correção de erros é assim chamado porque a variação de $X_{t}$ é explicada por dois componentes: os fatores de curto prazo, $\sum_{i=1}^{p-1} \Lambda_{i} \Delta X_{t-i}$; e a relação de longo prazo dada entre as coordenadas do vetor de variáveis endógenas, $\Phi X_{t-1}$, caso ocorra cointegração.

Um dos métodos para verificar a existência de cointegração é o teste de Johansen (1988), uma alternativa ao teste de Engle e Granger (1987). Johansen propõe definir o posto da matriz $\Phi$, da Equação (5),

$$
\Delta X_{t}=\Phi X_{t-1}+\sum_{i=1}^{p-1} \Lambda_{i} \Delta X_{t-i}+e_{t}
$$

e, dessa forma, estimar os vetores de cointegração $\beta$. Para definir o posto da matriz $\Phi$, Johansen sugeriu dois testes baseados em uma estimação de máxima verossimilhança com restrição.

Tem-se que $\Phi$ é uma matriz $n \times n$, e seu posto é dado por $r<n$, caso haja cointegração. Sendo o posto dessa matriz igual a $n$, as variáveis endógenas são todas estacionárias. Se o posto da matriz for nulo $(\Phi(I)=0)$, não há cointegração e as variáveis são não estacionárias. Como o determinante de uma matriz é o produto de seus autovalores, e considerando que o posto de $\Phi$ está entre zero e $n$, pode-se dizer 
que $\Phi$ terá $r$ autovalores diferentes de zero e $n-r$ autovalores iguais a zero. A ideia, então, é encontrar esses autovalores.

Variáveis determinísticas podem interferir nos valores críticos do modelo. Logo, incluem-se essas variáveis em $X_{t}$, no nível de vetor de cointegração, de modo que,

$$
X_{t}=\Phi_{1} X_{t-1}+\Phi_{2} X_{t-2}+\Phi_{p} X_{t-p}+\delta^{\prime} d_{t}+e_{t}
$$

em que: $d_{t}=[1, t]^{\prime}$ é um vetor com variáveis determinísticas, tais como dummies sazonais, entre outras; e, $\delta$, matriz de coeficientes de dimensão compatível com $d_{t}$, que, nesse caso, tem dimensão dada por $2 \times n$.

Na forma de VECM, o modelo anterior fica da seguinte forma:

$$
\Delta X_{t}=\Phi X_{t-1}+\sum_{i=1}^{p-1} \Lambda_{i} \Delta X_{t-i}+\delta^{\prime} d_{t}+e_{t}
$$

Para encontrar os autovalores da matriz $\Phi$, maximiza-se a Equação (20) com restrições sobre a matriz de covariância. Os autovalores são, então, ordenados do maior para o menor, $\lambda_{1}>\lambda_{2}>\cdots>\lambda_{n}$, sendo que cada um está associado a um autovetor que será associado aos vetores de cointegração contidos em $\beta$.

Nesse contexto, o primeiro teste proposto por Johansen é o teste de traço. A hipótese nula é de existência de $r^{*}$ vetores de cointegração. A hipótese alternativa é de $r>r^{*}$ vetores. De maneira formal, tem-se:

$$
\begin{aligned}
& H_{0}: r=r^{*} \\
& H_{1}: r>r^{*}
\end{aligned}
$$

A estatística de teste é dada por6:

$$
\lambda_{t r}(r)=-T \sum_{i=r+1}^{n} \ln \left(1-\hat{\lambda}_{i}\right) .
$$

0 posto da matriz $\Phi$ equivale ao número de suas raízes características, que são diferentes de zero. Caso não haja cointegração, os autovalores serão próximos de zero, demonstrando não estacionariedade ou instabilidade da matriz $\Phi$, e $\ln \left(1-\hat{\lambda}_{i}\right) \rightarrow 0$. Isso implica em pequenos valores para a estatística de traço, estabelecendo a não rejeição da hipótese nula. Se o $\hat{\lambda}_{i}$ é significantemente diferente de zero, então $\ln \left(1-\hat{\lambda}_{i}\right)$ será negativo. Logo, o valor da estatística de traço será alto, implicando na rejeição da hipótese.

0 teste é crescente, isto é, inicia-se com a soma de $n$ "logs" de 1 menos o autovalor, considerando, primeiramente, $r^{*}=0$. A rejeição da hipótese nula implica

6 Os autovalores são normalizados para que sempre sejam menores do que um. Logo, o ln será negativo. Isso é possível devido a não identificabilidade da matriz $\Phi$. 
a existência de mais de um vetor de cointegração. Continuando, parte-se para a soma dos $n-1$ "logs" de 1 menos os autovalores correspondentes, até o momento em que a hipótese nula não for mais rejeitada. Mackinnon, Haung e Michelis (1999) apresentam a tabela mais recente desse teste.

0 segundo teste é denominado de teste de autovalor e tende a apresentar resultados mais robustos que o teste de traço. A hipótese nula estabelece a existência de $r^{*}$ vetores de cointegração. Já a hipótese alternativa é de que existem $r^{*}+1$ vetores de cointegração. Formalmente,

$$
\begin{gathered}
H_{0}: r=r^{*} \\
H_{1}: r>r^{*}+1
\end{gathered}
$$

A estatística de teste é representada pela Equação (22):

$$
L R(r)=-T \ln \left(1-\hat{\lambda}_{r+1}\right)
$$

Em síntese, o teste de autovalor verifica qual o máximo autovalor significativo que dá origem a um vetor de cointegração. 0 teste também é crescente; rejeitar a hipótese nula significará que existe mais de um vetor de cointegração. Caso a hipótese nula não seja rejeitada, há $r^{*}$ vetores de cointegração. Vale lembrar que na realização do teste é preciso estabelecer cuidadosamente o número de defasagens. Além disso, o modelo deve ser determinado com cautela, levando em conta a existência de constante e tendência no nível do vetor $X_{t}$ e no vetor de cointegração.

\section{Resultados e discussões}

Esta seção se divide da seguinte forma: na primeira parte são apresentadas as variáveis; na segunda, os gráficos das séries e as estatísticas descritivas; na terceira, apresentam-se os testes de raiz unitária; a quarta demonstra os testes de cointegração; e, na quinta parte, faz-se o teste de causalidade de Granger/Bloco de exogeneidade e a análise das funções impulso resposta e decomposição da variância do erro de previsão.

\subsection{Apresentação das variáveis}

Este estudo compreendeu o período entre janeiro de 2011 e julho de $2016^{7}$, utilizando dados diários e perfazendo um total de 1.287 observações. Os índices financeiros foram coletados no site Yahoo! Finance e estão descritos na Tabela 1. O Índice

7 A escolha do período se justifica por evitar possíveis quebras estruturais causadas pela crise do subprime. Não é objeto deste estudo, no entanto, em trabalhos futuros, pretende-se considerar o período da crise do subprime e o anterior a ela, a fim de verificar se os resultados são mantidos. 
da Bolsa de Valores de São Paulo (IBOVESPA) é um indicador do desempenho médio das cotações dos ativos de maior negociabilidade e representatividade do mercado de ações brasileiro. No que se refere ao Índice de Consumo (ICON), este representa o desempenho médio das cotações dos ativos de maior negociabilidade e representatividade dos setores de consumo cíclico, consumo não cíclico e saúde. Em relação ao Índice de Energia Elétrica (IEE), o objetivo é ser o indicador do desempenho médio das cotações dos ativos de maior negociabilidade e representatividade desse setor. Já o Índice Financeiro (IFNC) tem por objetivo verificar o desempenho médio das cotações dos ativos de maior negociabilidade e representatividade dos setores de intermediários financeiros, serviços financeiros diversos, previdência e seguros. 0 Índice do Setor Industrial (INDX) mede o desempenho médio das cotações dos ativos de maior negociabilidade e representatividade dos setores da atividade industrial compreendidos por materiais básicos, bens industriais, consumo cíclico, consumo não cíclico, tecnologia da informação e saúde. Por fim, o Índice de Materiais Básicos (IMAT) serve como indicador do desempenho médio das cotações dos ativos de maior negociabilidade e representatividade do setor de materiais básicos ${ }^{8}$.

Tabela 1 - Variáveis, unidades, siglas e fontes

\begin{tabular}{cccc}
\hline Variável & Unidad & Sigla & Fonte \\
\hline Índice da Bolsa de Valores de São Paulo & Índice & IBOVESP & Yahoo!Financ \\
Índice de Consumo & Índice & ICON & Yahoo!Financ \\
Índice de Energia Elétrica & Índice & IEE & Yahoo!Financ \\
Índice do Setor Financeiro & Índice & IFNC & Yahoo!Financ \\
Índice do Setor Industrial & Índice & INDX & Yahoo!Financ \\
Índice de Materiais Básicos & Índice & IMAT & Yahoo!Financ \\
\hline
\end{tabular}

Fonte: elaboração própria.

\subsection{Gráficos das variáveis e estatísticas descritivas}

A Figura 1 apresenta a evolução de cada série de dados (variáveis em nível) ao longo do período de análise. Já a Tabela 2, apresenta as estatísticas descritivas básicas dos retornos diários dos índices, calculados da seguinte forma: $X_{t}=\ln \left(Y_{t}\right)-$ $\ln \left(Y_{t-1}\right)$, onde $Y_{t}$ é o vetor de índices diários. Vale dizer que, como os índices acionários são geralmente não estacionários, eles são frequentemente transformados em retornos. Os testes de raiz unitária estão descritos na subseção 4.3.

8 As informações deste parágrafo foram retiradas do site da BM\&FBOVESPA

(http://www.bmfbovespa.com.br/pt_br/produtos/indices/). 
Especificamente, em relação à Tabela 2, para diversos retornos as distribuições parecem ser assimétricas, uma vez que existem estimativas positivas e negativas de assimetria. Todas as séries de retornos possuem caudas pesadas e mostram um forte desvio da normalidade (os coeficientes de assimetria e curtose são todos diferentes daqueles da distribuição normal padrão, que são 0 e 3, respectivamente). Além disso, o teste de Jarque-Bera (JB) rejeitou a hipótese nula de normalidade ao nível de significância de 5\%. De acordo com Maldelbrot (1963) e Fama (1965), o excesso de curtose e a não normalidade são fatos estilizados no que se refere aos retornos financeiros.

Figura 1: Evolução das variáveis (em nível) no período de janeiro de 2011 a julho de 2016
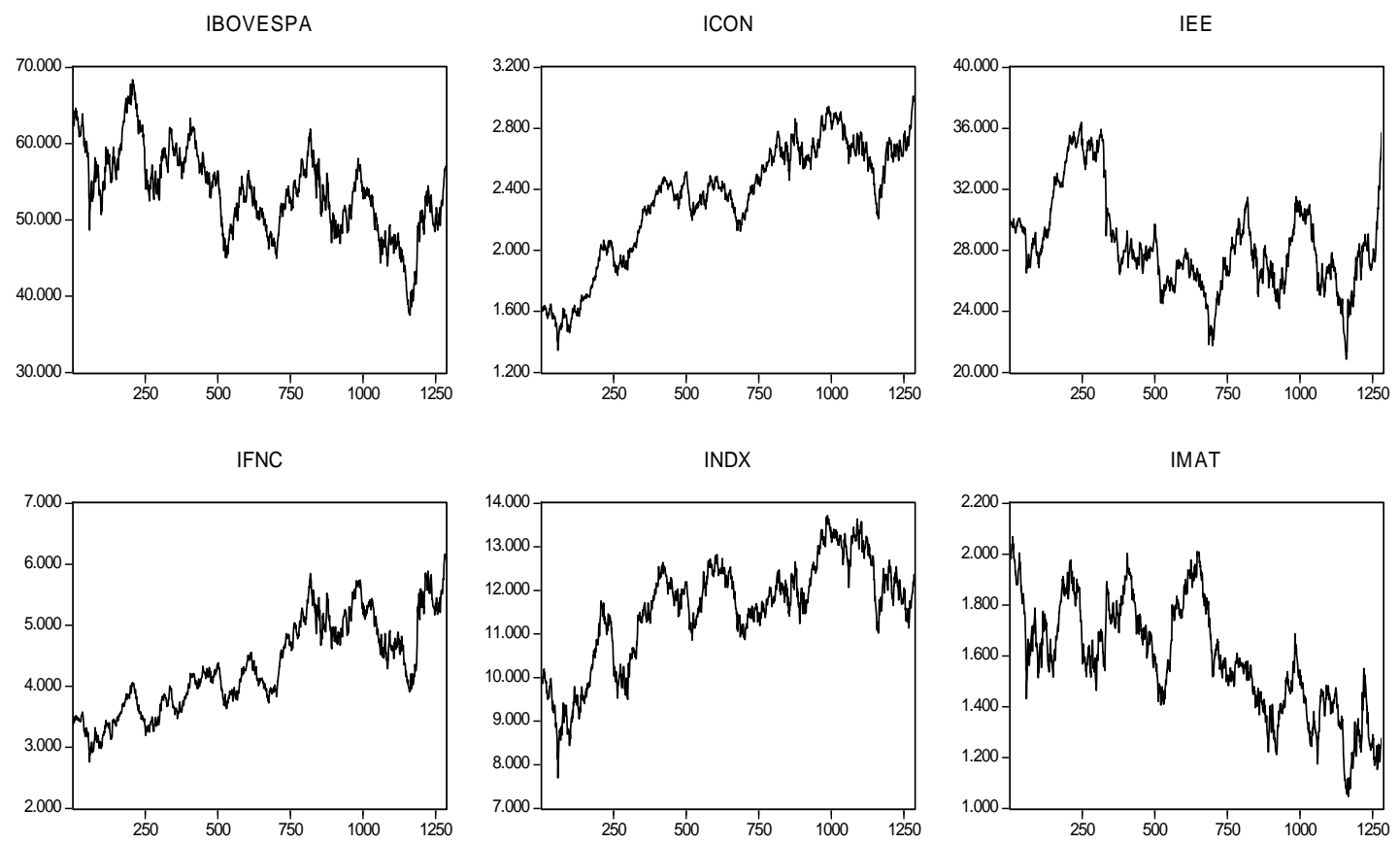

Fonte: elaboração própria a partir dos dados da pesquisa. 
Tabela 2: Estatísticas descritivas dos retornos diários

\begin{tabular}{lccccccccc}
\hline Variável & Média & Mediana & Máximo & Mínimo & $\begin{array}{l}\text { Desvio- } \\
\text { padrão }\end{array}$ & Assimetria & Curtose & Jarque-Bera & Valor-p \\
\hline DLIBOVESPA & $-0,00008$ & $-0,00054$ & 0,06387 & $-0,08431$ & 0,01529 & 0,04298 & 4,27796 & 87,9068 & 0,00000 \\
DLICON & 0,00047 & 0,00055 & 0,05202 & $-0,06240$ & 0,01120 & $-0,07313$ & 4,65518 & 147,9435 & 0,00000 \\
DLIEE & 0,00015 & 0,00029 & 0,04995 & $-0,08526$ & 0,01289 & $-0,44920$ & 5,64746 & 418,8141 & 0,00000 \\
DLIFNC & 0,00045 & $-0,00024$ & 0,09308 & $-0,08086$ & 0,01644 & 0,23745 & 5,20633 & 272,9211 & 0,00000 \\
DLINDX & 0,00016 & 0,00000 & 0,05248 & $-0,07368$ & 0,01182 & $-0,06073$ & 5,32143 & 289,5540 & 0,00000 \\
DLIMAT & $-0,00033$ & $-0,00066$ & 0,06936 & $-0,09071$ & 0,01792 & 0,10941 & 4,43172 & 112,4026 & 0,00000 \\
\hline
\end{tabular}

Fonte: elaboração própria a partir dos dados da pesquisa.

Notas: 1) D = significa a primeira diferença da variável; e 2) L = indica que as variáveis estão expressas em logaritmos.

\subsection{Testes de raiz unitária}

Vale destacar que todos os procedimentos econométricos foram realizados com as variáveis expressas em logaritmos naturais, a fim de estabilizar a variância dos índices em análise. Um dos primeiros passos na análise de séries temporais é a verificação da estacionariedade das séries. Caso não sejam estacionárias, devese realizar algum procedimento para estacionarizá-las (em geral, aplica-se a primeira diferença nas mesmas, dado que a maioria das séries econômicas é $I(1)$, ou seja, integradas de primeira ordem).

Neste trabalho, foram utilizados os seguintes testes de raiz unitária: Augmented Dickey-Fuller - ADF (DICKEY; FULLER, 1981); Phillips-Perron - PP (PHILLIPS; PERRON, 1988); e, Kwiatkowski-Phillips-Schmidt-Shin - KPSS (KWIATKOWSKI et al., 1992) ${ }^{9}$. Na realização do teste ADF foi utilizado o critério de informação de Schwarz (SIC), com o número de defasagens escolhido de forma automática e um máximo de 22 defasagens. No caso do teste PP, adotou-se o método de estimação espectral de Barllet Kernel e o critério de seleção de defasagens automático de Newey-West Bandwidth. Para o teste KPSS também foram utilizados a estimação espectral de Barllet Kernel e o critério Newey-West Bandwidth. A Tabela 3 apresenta os resultados dos referidos testes, que demonstram que todos os índices foram não estacionários em nível. Ressalta-se

9 Vale ressaltar que o teste KPSS é um teste assintótico, e deve ser utilizado em complemento aos demais testes de raiz unitária (BUENO, 2011). Assim, as conclusões sobre a existência de raiz unitária foram feitas primeiramente tomando como base os testes ADF e PP; em caso de divergência entre os testes ADF e PP, utilizou-se o teste KPSS como critério de desempate. Como critério de desempate também foram analisados os gráficos e os correlogramas (funções de autocorrelação) das séries. 
que uma vez aplicada a primeira diferença nas séries em logaritmos naturais, as mesmas tornaram-se estacionárias. Ademais, cabe dizer que não existe um consenso na literatura em relação a se trabalhar com as variáveis no modelo VAR em nível e/ou em primeira diferença. Sims (1980) e Sims, Stock e Watson (1990), por exemplo, não encontram grandes problemas em se trabalhar com variáveis estacionárias e não estacionárias em um modelo VAR.

Tabela 3: Testes de raiz unitária para as variáveis em nível

\begin{tabular}{|c|c|c|c|c|c|c|c|c|c|}
\hline Variável & $\mathrm{ADF}$ & $\mathrm{K}$ & & PP & $\mathrm{K}$ & & KPSS & $\mathrm{K}$ & \\
\hline LIBOVESPA & $-3,1196 \mathrm{~ns}$ & 0 & (ct) & $-3,0673^{\mathrm{ns}}$ & 5 & (ct) & $0,0829 * * *$ & 29 & (ct) \\
\hline LICON & $-2,2853 \mathrm{~ns}$ & 0 & (ct) & $-2,2144 \mathrm{~ns}$ & 11 & (ct) & $0,6513^{* * *}$ & 29 & (ct) \\
\hline LIEE & $-1,8594 \mathrm{~ns}$ & 1 & (cc) & $-1,8287 \mathrm{~ns}$ & 8 & (cc) & $0,2675^{* * *}$ & 29 & (ct) \\
\hline LIFNC & $-2,8956 \mathrm{~ns}$ & 0 & (ct) & $-3,3118^{*}$ & 7 & (ct) & $0,2085^{* *}$ & 29 & (ct) \\
\hline LINDX & $-2,9096 \mathrm{~ns}$ & 0 & (ct) & $-2,8225^{n s}$ & 3 & (ct) & $0,4482^{* * *}$ & 29 & (ct) \\
\hline LIMAT & $-2,4333 \mathrm{~ns}$ & 0 & (ct) & $2,5022 \mathrm{~ns}$ & 2 & (ct) & $0,3184^{* * *}$ & 29 & (ct) \\
\hline
\end{tabular}

Fonte: elaboração própria a partir dos dados da pesquisa.

Nota: 1) *** Significativo a $1 \%$, ** Significativo a $5 \%$, * Significativo a $10 \%$, ns Não significativo a $10 \% ; 2$ )

$\mathrm{K}=$ número de defasagens de cada teste para cada variável; 3) $\mathrm{L}=$ indica que as variáveis estão expressas em logaritmos; e, 4) (ct) representa com constante e com tendência e (cc) com constante.

\subsection{Teste de cointegração de Johansen}

Uma vez realizados os testes de raiz unitária, apresentam-se os testes de cointegração de Johansen. É válido mencionar que na realização do teste de cointegração adotou-se o logaritmo das variáveis em nível (e não em primeira diferença), conforme determinado pela literatura. Na elaboração do teste é fundamental determinar o número de defasagens. Para isso, estimou-se um VAR irrestrito, observando os critérios do erro de previsão final (FPE), de Akaike (AIC), de Schwarz (SC) e de Hannan-Quinn (HQ) para selecionar o número de defasagens a ser empregado. $\mathrm{O}$ critério $\mathrm{HQ}$ sugeriu a utilização de uma defasagem para o modelo, conforme a Tabela 4. Já os critérios FPE, AIC e SC indicaram a adoção de duas defasagens. No entanto, optou-se por trabalhar com cinco defasagens, uma vez que os modelos com duas, três e quatro defasagens apresentaram autocorrelação dos resíduos em algumas defasagens e heteroscedasticidade. 
Tabela 4: Determinação do número de defasagens do modelo VAR

\begin{tabular}{ccccc}
\hline Defasagem & FPE & AIC & SC & HQ \\
\hline 0 & $3,49 \mathrm{E}-14$ & $-13,95923$ & $-13,93505$ & $-13,95015$ \\
1 & $5,63 \mathrm{E}-26$ & $-41,11198$ & $-40,9427^{*}$ & $-41,0484^{*}$ \\
2 & $5,53 \mathrm{e}-26^{*}$ & $-41,1302^{*}$ & $-40,8159$ & $-41,01219$ \\
3 & $5,63 \mathrm{E}-26$ & $-41,11193$ & $-40,65256$ & $-40,93943$ \\
4 & $5,72 \mathrm{E}-26$ & $-41,09519$ & $-40,49075$ & $-40,86822$ \\
5 & $5,83 \mathrm{E}-26$ & $-41,07618$ & $-40,32668$ & $-40,79474$ \\
6 & $5,96 \mathrm{E}-26$ & $-41,05515$ & $-40,16059$ & $-40,71924$ \\
7 & $6,13 \mathrm{E}-26$ & $-41,02733$ & $-39,98770$ & $-40,63694$ \\
8 & $6,29 \mathrm{E}-26$ & $-41,00165$ & $-39,81696$ & $-40,55679$ \\
\hline
\end{tabular}

Fonte: elaboração própria a partir dos dados da pesquisa.

Nota: 1) * Indica a ordem selecionada pelo critério; e, 2) FPE = erro de previsão final; AIC = Akaike, SC = Schwarz e HQ = Hannan-Quinn.

Nas TABELAS 5 e 6 são apresentados os testes de traço $\left(\lambda_{t r}\right)$ e máximo autovalor $\left(L R_{\max }\right)$. Como pode ser observado, ambos os testes revelaram que não existe vetor de cointegração entre os índices considerados neste estudo, demonstrando que não há uma relação de longo prazo entre as variáveis LIBOVESPA, LICON, LIEE, LIFNC, LINDX e LIMAT. Isso pode ser decorrente da grande absorção das informações que existem no mercado financeiro.

Tabela 5: Resultados do teste de traço

\begin{tabular}{ccccc}
\hline$H_{0}$ & $H_{1}$ & $\lambda_{t r}$ & $\lambda_{t r}$ (crítico) & Valor-p \\
\hline$r=0$ & $r>0$ & 69,5182 & 95,7536 & $0,7384^{\text {ns }}$ \\
$r \leq 1$ & $r>1$ & 42,1871 & 69,8189 & $0,9071^{\text {ns }}$ \\
$r \leq 2$ & $r>2$ & 22,6060 & 47,8561 & $0,9670^{\text {ns }}$ \\
\hline
\end{tabular}

Fonte: Elaboração própria, a partir dos dados da pesquisa.

Nota: ${ }^{\text {ns }}=$ não significativo a $10 \%$.

Tabela 6 - Resultados do teste de máximo autovalor

\begin{tabular}{ccccc}
\hline$H_{0}$ & $H_{1}$ & $L R_{\max }$ & $L R_{\max }$ (crítico) & Valor-p \\
\hline$r=0$ & $r=1$ & 27,3311 & 40,0776 & $0,6096^{\text {ns }}$ \\
$r=1$ & $r=2$ & 19,5810 & 33,8769 & $0,7850^{\text {ns }}$ \\
$r=2$ & $r=3$ & 11,0119 & 27,5843 & $0,9651^{\text {ns }}$ \\
\hline
\end{tabular}

Fonte: elaboração própria a partir dos dados da pesquisa. Nota: ns = não significativo a $10 \%$. 


\subsection{Vetor autorregressivo}

Dado que o $\operatorname{posto}(\Phi)=0$, ou seja, as séries não são cointegradas, deve-se estimar o VAR com as séries em primeiras diferenças: DLIBOVESPA, DLICON, DLIEE, DLIFNC, DLINDX e DLIMAT. Como o melhor VAR irrestrito é aquele com cinco defasagens, o VAR com as séries em primeira diferença foi estimado com quatro defasagens. 0 modelo apresentou todas as raízes do polinômio dentro do círculo unitário, satisfazendo a condição de estabilidade. Além disso, os resultados foram satisfatórios para não autocorrelação (utilizou-se de uma a 12 defasagens). No que tange ao teste de normalidade dos resíduos, o teste de Jarque-Bera rejeitou a hipótese nula de que os resíduos sejam normais. Esse resultado já era esperado devido às características de volatilidade das séries financeiras. Ressalta-se que a rejeição da normalidade não impede a interpretação ou a análise dos resultados do modelo, em função do grande número de observações que compõem a amostra. Destaca-se que, ainda, que Oreiro et al. (2006) salientam que esse procedimento é comum em alguns trabalhos no Brasil, como Grôppo (2004) e Camuri (2005).

Antes de demonstrar as funções de impulso-resposta e a decomposição da variância do erro de previsão, e por se tratar de um modelo multivariado, são apresentados os resultados do teste de causalidade de Granger/Bloco de exogeneidade, que verifica se uma variável endógena pode ser tratada como exógena (Tabela 7). Para cada equação do modelo VAR, pela estatística Wald, testa-se a significância de cada uma das outras variáveis endógenas desfasadas na equação. Pelo teste de causalidade de Granger, pode-se verificar as relações causalidade entre as varáveis, no sentido "Granger causa".

i) Causalidade bidirecional:

- $\quad$ DLIBOVESPA $\leftrightarrow$ DLICON;

- $\quad$ DLIBOVESPA $\leftrightarrow$ DLIFNC;

- $\quad$ DLIBOVESPA $\leftrightarrow$ DLINDX;

- $\quad$ DLIBOVESPA $\leftrightarrow$ DLIMAT;

- DLINDX $\leftrightarrow$ DLIFNC.

ii) Causalidade unidirecional:
- $\quad$ DLINDX $\rightarrow$ DLICON;
- $\quad$ DLICON $\rightarrow$ DLIMAT;
- $\quad$ DLINDX $\rightarrow$ DLIEE;
- $\quad$ DLIMAT $\rightarrow$ DLIFNC;
- DLINDX $\rightarrow$ DLIMAT. 
Tabela 7 - Causalidade de Granger/Bloco de exogeneidade

\begin{tabular}{ccccccc}
\hline \multicolumn{7}{c}{ Variável dependente } \\
\hline Não causa & DLIBOVESPA & DLICON & DLIEE & DLIFNC & DLINDX & DLIMAT \\
\hline DLIBOVESPA & - & $8,2721^{* *}$ & $6,8838^{\mathrm{ns}}$ & $8,6715^{*}$ & $12,4741^{* *}$ & $11,2474^{* *}$ \\
DLICON & $8,0549^{*}$ & - & $3,7166^{\mathrm{ns}}$ & $7,085^{\mathrm{ns}}$ & $5,0034^{\mathrm{ns}}$ & $7,5672^{*}$ \\
DLIEE & $2,6826^{\mathrm{ns}}$ & $1,8717^{\mathrm{ns}}$ & - & $1,9909^{\mathrm{ns}}$ & $2,125^{\mathrm{ns}}$ & $3,5405^{\mathrm{ns}}$ \\
DLIFNC & $7,7648^{*}$ & $3,2451^{\mathrm{ns}}$ & $5,0236^{\mathrm{ns}}$ & - & $7,9212^{*}$ & $4,4611^{\mathrm{ns}}$ \\
DLINDX & $13,7961^{* * *}$ & $13,2364^{* *}$ & $7,6219^{*}$ & $12,3333^{* *}$ & - & $15,5022^{* * *}$ \\
DLIMAT & $9,7269^{* *}$ & $6,1164^{\mathrm{ns}}$ & $6,8668^{\mathrm{ns}}$ & $11,3217^{* *}$ & $4,9415^{\mathrm{ns}}$ & - \\
Todos & $32,9955^{* *}$ & $30,5632^{*}$ & $23,266^{\mathrm{ns}}$ & $28,6313^{*}$ & $25,1248^{\mathrm{ns}}$ & $37,5239^{* *}$ \\
\hline
\end{tabular}

Fonte: elaboração própria, a partir dos dados da pesquisa.

Nota: 1) *** Significativo a 1\%, ** Significativo a $5 \%$, * Significativo a $10 \%$, ns = não significativo a $10 \%$;

"Todos" indica o teste de causalidade para o conjunto de todas as variáveis independentes.

Finalizada a etapa de identificação e estimação do VAR, serão analisadas as funções de impulso-resposta. Para estimação das funções de impulso-reposta foi utilizado o método da função de impulso generalizado, que elimina o problema relacionado à ordenação das variáveis no modelo VAR. Contrário ao denominado método de ordenamento de Cholesky, que utiliza a "hipótese da ortogonalidade", o método de impulso resposta generalizado não varia caso ocorra uma reordenação das variáveis no VAR (LÜTKEPOHL, 1991; KOOP; PESARAN; POTTER, 1996; PESARAN; SHIN, 1998). Ressalta-se ainda, que conforme Edwing (2003), o método generalizado apresenta duas principais vantagens: a) os resultados da função impulso-resposta generalizada apresentam maior robustez do que o método ortogonalizado; e b) uma vez que a ortogonalidade não é imposta, a função de impulso-reposta generalizada possibilita uma interpretação com maior acurácia a resposta do efeito inicial a um choque causada por uma variável sobre as demais variáveis consideradas no modelo estimado.

Na Figura 2, são demonstradas as funções de impulso-resposta considerando os efeitos dos índices setoriais sobre o IBOVESPA (DLIBOVESPA). Observa-se que todos os índices setoriais têm impactos significativos sobre o índice global IBOVESPA, sendo que os maiores efeitos foram causados por DLIFNC, DLINDX e DLICON, respectivamente. Vale lembrar que, as empresas desses três setores têm grande peso na formação do IBOVESPA, especialmente os setores financeiro e industrial. Para exemplificar a análise de uma função de impulso-resposta, toma-se o caso do efeito do índice setorial DLIFNC sobre a variável DLIBOVESPA. Verifica-se que uma elevação de um desvio-padrão em DLIFNC causou um efeito positivo sobre DLIBOVESPA no 
primeiro dia após o choque e de maneira relativamente alta. Além disso, observa-se que após o segundo dia, a tendência foi de estabilidade, isto é, os efeitos de DLIFNC sobre DLIBOVESPA foram dissipados rapidamente, retornando ao patamar anterior ao choque. Cabe dizer que em todos os casos, não existiu continuidade dos choques. Os efeitos de choques nos índices setoriais sobre o IBOVESPA foram dissipados rapidamente em função da dinâmica e da grande absorção das informações que existe no mercado financeiro.

Figura 2: Funções de impulso-resposta generalizadas considerando os efeitos dos índices setoriais sobre IBOVESPA (DLIBOVESPA)

Response to Generalized One S.D. Innovations \pm 2 S.E.

Response of DLIBOVESPA to DLIBOVESPA

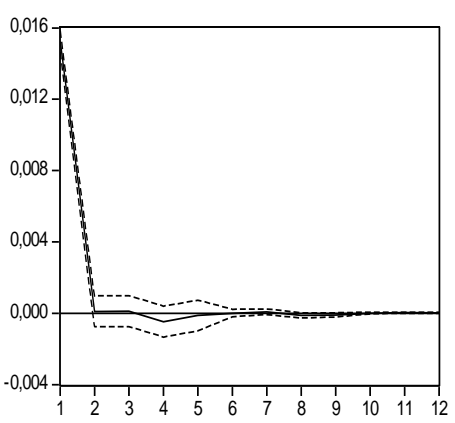

Response of DLIBOVESPA to DLIFNC

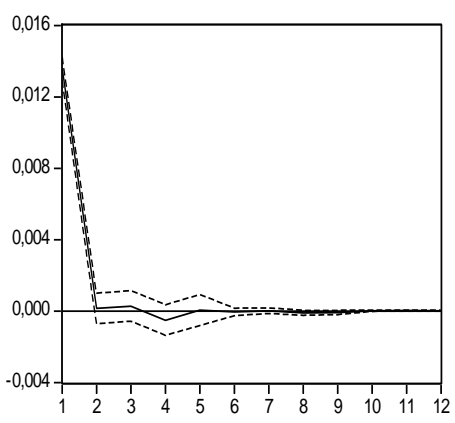

Response of DLIBOVESPA to DLICON

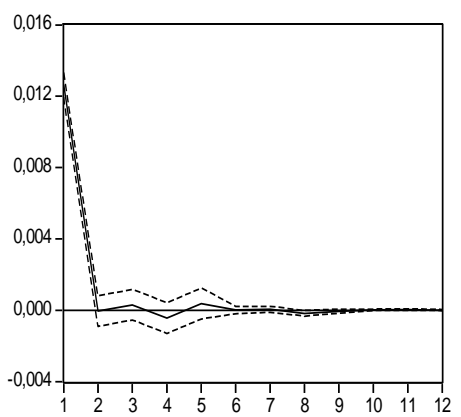

Response of DLIBOVESPA to DLINDX

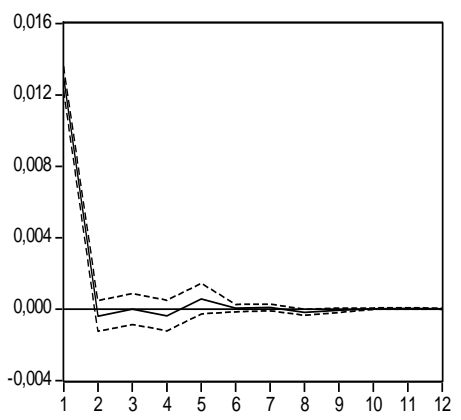

Response of DLIBOVESPA to DLIEE

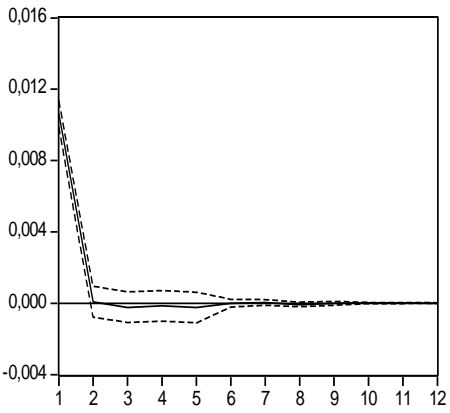

Response of DLIBOVESPA to DLIMAT

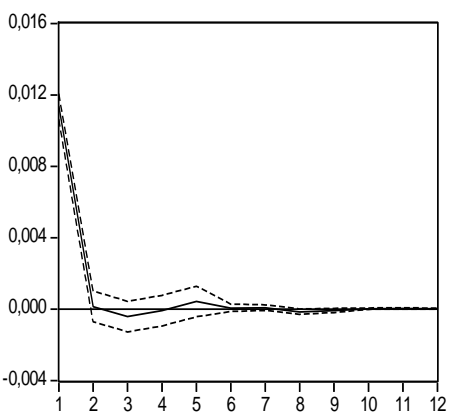

Fonte: elaboração própria a partir dos dados da pesquisa.

Notas: 1) $\mathrm{D}$ = significa a primeira diferença da variável; 2) $\mathrm{L}$ = indica que as variáveis estão expressas em logaritmos; e, 3) Response of " $A$ " to " $B$ " = Resposta de " $A$ " à " $B$ ". 
A fim de corroborar a inter-relação que existe entre os índices financeiros considerados na pesquisa, as Figuras 3 e 4 apresentam as funções de impulsoresposta generalizadas considerando os efeitos de choques em DLIFNC e DLINDX, respectivamente, sobre os demais índices considerados ${ }^{10}$. Conforme pode ser observado, além do forte efeito sobre o IBOVESPA, os índices dos setores financeiro e industrial apresentaram efeitos relevantes e significantes sobre os demais setores em estudo, demonstrando a forte inter-relação no mercado financeiro (acionário). Assim, choques que ocorrem em um setor (índice) tendem a se propagar para os índices de outros setores. Para exemplificar, choques no mercado financeiro tendem a impactar significativamente o lado real da economia. Crises no mercado financeiro podem reduzir os financiamentos bancários, aumentar os custos da tomada de crédito, criar dificuldades no mercado de capitais, reduzir o nível de poupança, entre outros. Esses reflexos são propagados para o lado real da economia, afetando o nível de atividade econômica e, consequentemente, as decisões de investimento (CASTRO; BRANDÃO, 2008). Por conseguinte, o setor industrial é afetado (índice do setor industrial).

Do mesmo modo, em todos os casos não existiu a continuidade dos choques. Os efeitos de choques em um índice sobre os demais foram dissipados rapidamente, devido à dinâmica e à grande absorção das informações que existe no mercado financeiro. No entanto, não se pode descartar a interdependência entre os índices em estudo (índice global IBOVESPA e índices setoriais). Mesmo não havendo cointegração, observa-se que houve influência entre as variáveis, revelando que os efeitos positivos ou negativos podem ser disseminados entre os índices analisados (efeito contágio), o que tem influência direta sobre as decisões de investimentos dos agentes econômicos e financeiros, especialmente no que tange à diversificação de suas carteiras de ativos.

10 Funções de impulso-reposta foram realizadas levando-se em conta choques nos demais índices setoriais, e podem ser consultadas junto ao autor. 
Figura 3: Funções de impulso-resposta generalizada considerando um choque em DLIFNC

Response to Generalized One S.D. Innovations \pm 2 S.E

Response of DLIBOVESPA to DLIFNC

Response of DLICON to DLIFNC

Response of DLIEE to DLIFNC
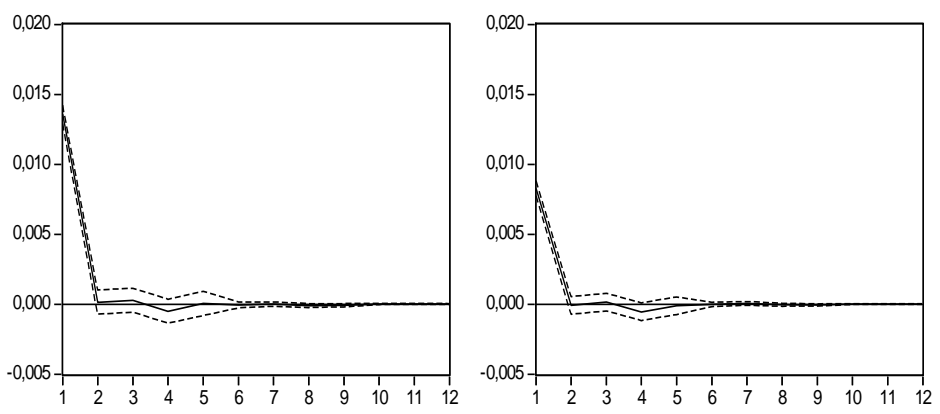

Response of DLIFNC to DLIFNC

Response of DLINDX to DLIFNC
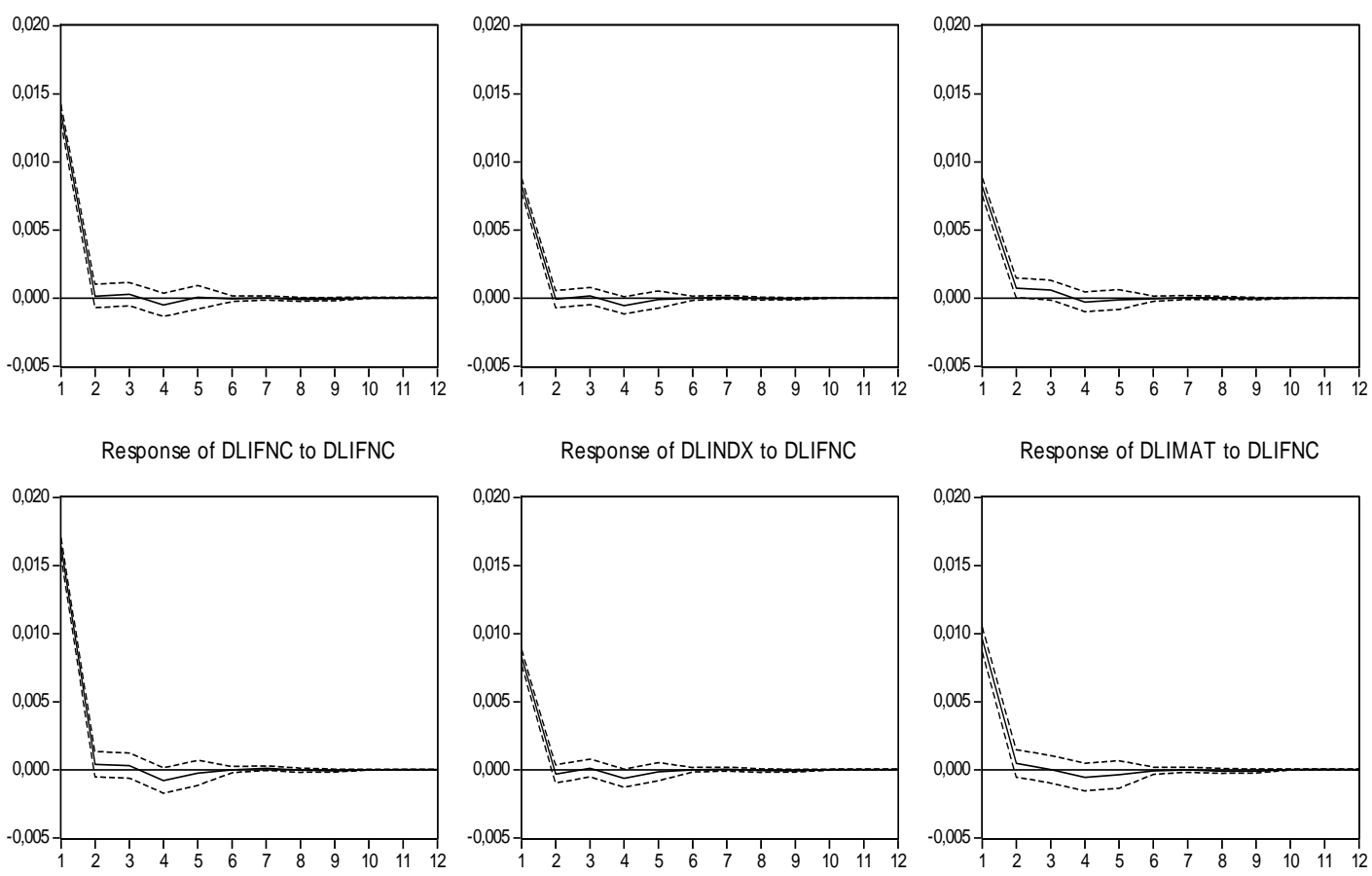

Response of DLIMAT to DLIFNC

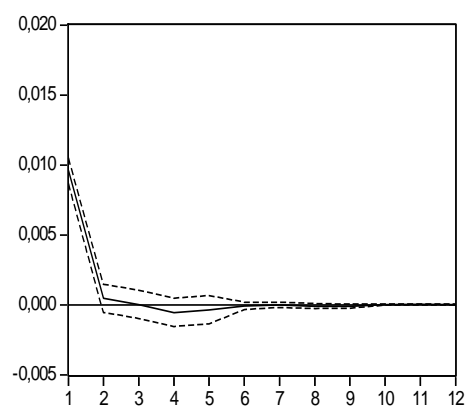

Fonte: elaboração própria, a partir dos dados da pesquisa.

Notas: 1) $\mathrm{D}$ = significa a primeira diferença da variável; 2) $\mathrm{L}$ = indica que as variáveis estão expressas em logaritmos; 3 ) Response of " $A$ " to " $B$ " = Resposta de " $A$ " a " $B$ ". 
Figura 4: Funções de impulso-resposta generalizada considerando um choque em DLINDX

Response to Generalized One S.D. Innovations \pm 2 S.E
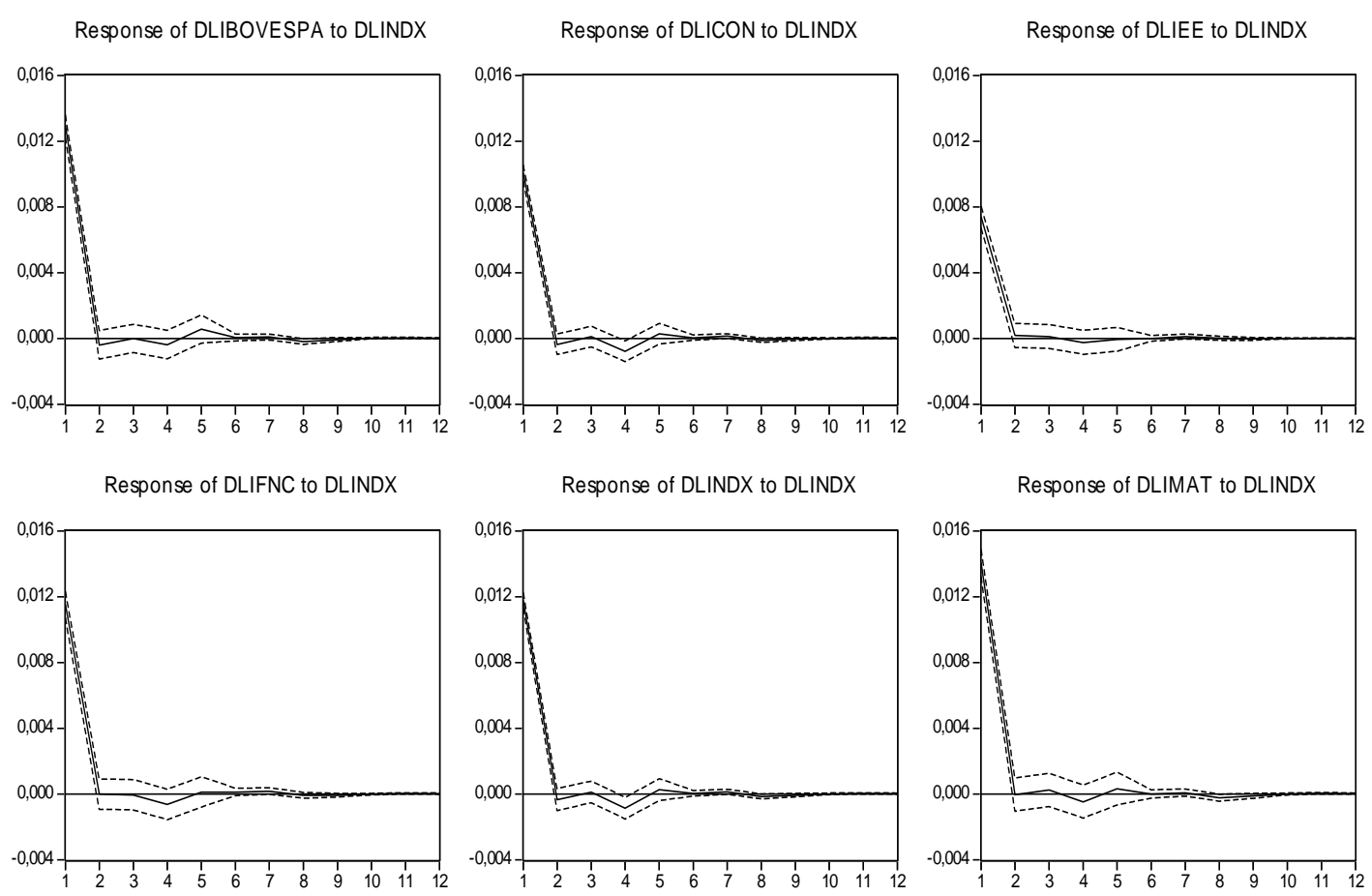

Fonte: elaboração própria, a partir dos dados da pesquisa.

Notas: 1) $\mathrm{D}$ = significa a primeira diferença da variável; 2) $\mathrm{L}$ = indica que as variáveis estão expressas em logaritmos; 3) Response of " $A$ " to " $B$ " = Resposta de "A" a "B".

A metodologia VAR permite, em complemento à análise das funções de impulsoresposta, a realização do exercício de decomposição da variância (DV), com o intuito de verificar a porcentagem da variância do erro de previsão, que é decorrente de cada variável endógena ao longo do horizonte de previsão. Ao tratar da decomposição da variância do erro de previsão, a questão referente à ordenação das variáveis também é de suma importância. No entanto, diferentemente do método generalizado, adotado para a estimação das funções de impulso-resposta, aqui se utilizou o ordenamento de Cholesky, uma vez que a fatoração não ortogonal produz decomposições que não satisfazem a propriedade de soma. Logo, para a decomposição da variância do erro de previsão, a fatoração é limitada às fatorações ortogonais. Nesse caso, baseando-se em conhecimentos prévios (teoria econômica, conhecimento do mercado, artigos, entre 
outros), a ordenação escolhida foi: DLIFNC, DLINDX, DLIMAT, DLICON, DLIEE e DLIBOVESPA.

Na Tabela 8 são apresentados os resultados da decomposição para a variável DLIBOVESPA. Nota-se que os maiores percentuais de explicação do erro de previsão do índice IBOVESPA são decorrentes do índice do setor financeiro (DLIFNC). Vale destacar que considerando a carteira teórica do IBOVESPA, para o dia 05 de setembro de 2017, aproximadamente 30\% do IBOVESPA era composto por papeis de empresas ligadas ao setor financeiro. 0 setor industrial (DLINDX) teve forte influência sobre o erro de previsão do IBOVESPA, sendo que a participação das empresas que compõem o índice industrial no índice IBOVESPA também é relevante. Além disso, cabe mencionar que não existem variações muito expressivas dos percentuais de explicação após o segundo período, mais uma vez demonstrando a grande absorção das informações que existe no mercado financeiro.

Tabela 8: Decomposição histórica da variância do erro de previsão da variável DLIBOVESPA

\begin{tabular}{cccccccc}
\hline Período & ERRO-PADRÃO & DLIBOVESPA & DLICON & DLIEE & DLIFNC & DLINDX & DLIMAT \\
\hline 1 & 0,0152 & 7,6333 & 0,6896 & 0,6776 & 79,3084 & 9,8187 & 1,8725 \\
2 & 0,0153 & 7,5571 & 1,2163 & 0,6734 & 78,5243 & 9,9306 & 2,0984 \\
3 & 0,0154 & 7,5516 & 1,2490 & 0,7977 & 78,2003 & 9,9194 & 2,2820 \\
4 & 0,0154 & 7,5720 & 1,2484 & 0,8188 & 78,1407 & 9,8981 & 2,3221 \\
5 & 0,0154 & 8,1199 & 1,2605 & 0,9231 & 77,3632 & 10,0325 & 2,3008 \\
6 & 0,0155 & 8,1202 & 1,2604 & 0,9231 & 77,3582 & 10,0369 & 2,3013 \\
7 & 0,0155 & 8,1260 & 1,2611 & 0,9234 & 77,3479 & 10,0407 & 2,3010 \\
8 & 0,0155 & 8,1267 & 1,2621 & 0,9238 & 77,3374 & 10,0495 & 2,3006 \\
9 & 0,0155 & 8,1282 & 1,2620 & 0,9256 & 77,3342 & 10,0489 & 2,3011 \\
10 & 0,0155 & 8,1284 & 1,2620 & 0,9256 & 77,3340 & 10,0489 & 2,3011 \\
11 & 0,0155 & 8,1284 & 1,2620 & 0,9257 & 77,3337 & 10,0490 & 2,3011 \\
12 & 0,0155 & 8,1288 & 1,2620 & 0,9257 & 77,3334 & 10,0490 & 2,3011 \\
\hline
\end{tabular}

Fonte: elaboração própria, a partir dos dados da pesquisa.

Notas: 1) D = significa a primeira diferença da variável; 2) $\mathrm{L}$ = indica que as variáveis estão expressas em logaritmos. 
Por fim, as Tabelas 9, 10 e 11 demostram a decomposição do erro de previsão para as variáveis DLIFNC, DLINDX e DLICON, respectivamente ${ }^{11}$. Observa-se que a variância do erro de previsão do índice do setor financeiro (DLIFNC) é quase autoexplicativa. Quanto ao erro de previsão do índice industrial, verifica-se que além de um forte efeito do próprio índice, o setor financeiro tem relevante influência sobre o mesmo, o que pode ser explicado, conforme descrito anteriormente, pelos reflexos do setor financeiro sobre o lado real da economia, especificamente, sobre o setor industrial. Outrossim, a variância do erro de previsão de DLICON é influenciada pelo próprio setor e pelos setores financeiro, industrial e de materiais básicos. Assim como no caso das funções de impulso-reposta, os resultados da decomposição da variância do erro de previsão revelaram que existe uma grande inter-relação entre os índices estudados, a saber: índice global IBOVESPA e índices setoriais.

Tabela 9: Decomposição histórica da variância do erro de previsão da variável DLIFNC

\begin{tabular}{cccccccc}
\hline Período & ERRO-PADRÃO & DLIBOVESPA & DLICON & DLIEE & DLIFNC & DLINDX & DLIMAT \\
\hline 1 & 0,0152 & 0,0000 & 0,0000 & 0,0000 & 100,0000 & 0,0000 & 0,0000 \\
2 & 0,0153 & 0,0002 & 0,5549 & 0,0029 & 99,1185 & 0,0578 & 0,2657 \\
3 & 0,0154 & 0,3177 & 0,6195 & 0,0447 & 98,4412 & 0,1084 & 0,4685 \\
4 & 0,0154 & 0,3167 & 0,6412 & 0,0446 & 98,3533 & 0,1125 & 0,5317 \\
5 & 0,0154 & 0,6471 & 0,6561 & 0,0445 & 97,9562 & 0,1666 & 0,5294 \\
6 & 0,0155 & 0,6496 & 0,6561 & 0,0453 & 97,9426 & 0,1767 & 0,5298 \\
7 & 0,0155 & 0,6517 & 0,6570 & 0,0454 & 97,9332 & 0,1830 & 0,5298 \\
8 & 0,0155 & 0,6567 & 0,6574 & 0,0454 & 97,9246 & 0,1858 & 0,5301 \\
9 & 0,0155 & 0,6567 & 0,6574 & 0,0459 & 97,9228 & 0,1869 & 0,5303 \\
10 & 0,0155 & 0,6571 & 0,6574 & 0,0459 & 97,9223 & 0,1870 & 0,5303 \\
11 & 0,0155 & 0,6574 & 0,6574 & 0,0459 & 97,9219 & 0,1871 & 0,5303 \\
12 & 0,0155 & 0,6574 & 0,6574 & 0,0460 & 97,9218 & 0,1871 & 0,5303 \\
\hline
\end{tabular}

Fonte: elaboração própria, a partir dos dados da pesquisa.

Notas: 1) D = significa a primeira diferença da variável; 2) L = indica que as variáveis estão expressas em logaritmos.

11 A decomposição da variância do erro de previsão para os demais índices pode ser consultada junto ao autor. 
Tabela 10: Decomposição histórica da variância do erro de previsão da variável DLINDX

\begin{tabular}{cccccccc}
\hline Período & ERRO-PADRÃO & DLIBOVESPA & DLICON & DLIEE & DLIFNC & DLINDX & DLIMAT \\
\hline 1 & 0,0152 & 0,0000 & 0,0000 & 0,0000 & 49,3250 & 50,6751 & 0,0000 \\
2 & 0,0153 & 0,0026 & 0,3053 & 0,0168 & 49,1559 & 50,4488 & 0,0706 \\
3 & 0,0154 & 0,0235 & 0,3200 & 0,0607 & 49,0818 & 50,3640 & 0,1500 \\
4 & 0,0154 & 0,0278 & 0,3192 & 0,1545 & 49,0342 & 50,2875 & 0,1769 \\
5 & 0,0154 & 0,9753 & 0,3172 & 0,2481 & 48,4258 & 49,8391 & 0,1945 \\
6 & 0,0155 & 0,9761 & 0,3172 & 0,2481 & 48,4241 & 49,8399 & 0,1947 \\
7 & 0,0155 & 0,9819 & 0,3175 & 0,2480 & 48,4144 & 49,8434 & 0,1947 \\
8 & 0,0155 & 0,9953 & 0,3181 & 0,2489 & 48,3991 & 49,8439 & 0,1947 \\
9 & 0,0155 & 0,9959 & 0,3183 & 0,2515 & 48,3989 & 49,8401 & 0,1954 \\
10 & 0,0155 & 0,9963 & 0,3183 & 0,2515 & 48,3987 & 49,8399 & 0,1954 \\
11 & 0,0155 & 0,9966 & 0,3183 & 0,2516 & 48,3983 & 49,8398 & 0,1954 \\
12 & 0,0155 & 0,9970 & 0,3183 & 0,2517 & 48,3982 & 49,8395 & 0,1954 \\
\hline
\end{tabular}

Fonte: elaboração própria, a partir dos dados da pesquisa.

Notas: 1) $\mathrm{D}$ = significa a primeira diferença da variável; 2) $\mathrm{L}$ = indica que as variáveis estão expressas em logaritmos.

Tabela 11 - Decomposição histórica da variância do erro de previsão da variável DLICON

\begin{tabular}{cccccccc}
\hline Período & ERRO-PADRÃO & DLIBOVESPA & DLICON & DLIEE & DLIFNC & DLINDX & DLIMAT \\
\hline 1 & 0,0152 & 0,0000 & 11,5719 & 0,0000 & 55,2262 & 28,7475 & 4,4544 \\
2 & 0,0153 & 0,0320 & 12,0994 & 0,0189 & 54,7726 & 28,6496 & 4,4275 \\
3 & 0,0154 & 0,1335 & 12,0851 & 0,0273 & 54,5702 & 28,5347 & 4,6492 \\
4 & 0,0154 & 0,1333 & 12,0671 & 0,1367 & 54,4029 & 28,5765 & 4,6836 \\
5 & 0,0154 & 0,6350 & 11,9637 & 0,2705 & 53,9455 & 28,5255 & 4,6598 \\
6 & 0,0155 & 0,6382 & 11,9630 & 0,2711 & 53,9416 & 28,5267 & 4,6595 \\
7 & 0,0155 & 0,6415 & 11,9607 & 0,2720 & 53,9319 & 28,5356 & 4,6585 \\
8 & 0,0155 & 0,6508 & 11,9586 & 0,2723 & 53,9200 & 28,5410 & 4,6573 \\
9 & 0,0155 & 0,6517 & 11,9580 & 0,2737 & 53,9196 & 28,5395 & 4,6576 \\
10 & 0,0155 & 0,6520 & 11,9580 & 0,2737 & 53,9193 & 28,5394 & 4,6576 \\
11 & 0,0155 & 0,6523 & 11,9578 & 0,2738 & 53,9189 & 28,5396 & 4,6576 \\
12 & 0,0155 & 0,6526 & 11,9578 & 0,2738 & 53,9188 & 28,5395 & 4,6575 \\
\hline
\end{tabular}

Fonte: elaboração própria, a partir dos dados da pesquisa.

Notas: 1) D = significa a primeira diferença da variável; 2) $\mathrm{L}$ = indica que as variáveis estão expressas em logaritmos. 


\section{Considerações finais}

Este estudo objetivou verificar as inter-relações entre o Índice da Bolsa de Valores de São Paulo (IBOVESPA) e os índices financeiros setoriais: Índice de Consumo (ICON); Índice de Energia Elétrica (IEE); Índice do Setor Financeiro (IFNC); Índice do Setor Industrial (INDX); e, Índice de Materiais Básicos (IMAT). Para isso, utilizou-se a metodologia VAR/VECM, considerando o período de janeiro de 2011 a julho de 2016.

No que se refere à análise de cointegração, os resultados revelaram que não existe vetor de cointegração entre os índices estudados, isto é, não há uma relação de longo prazo entre as variáveis: IBOVESPA, ICON, IEE, IFNC, INDX e IMAT. Uma vez que não existe tal relação, um modelo vetorial autorregressivo (VAR) foi estimado. A partir desse modelo foram estimadas as funções de impulso resposta e foi realizada a análise de decomposição da variância do erro de previsão. Por meio das análises, verificou-se que existe uma forte inter-relação entre os índices considerados no estudo, a saber: o índice global IBOVESPA e os índices setoriais. Além disso, observouse que os efeitos de um índice sobre os demais são dissipados rapidamente, dada a dinâmica e a grande absorção das informações que existe no mercado financeiro.

Por fim, cabe destacar que a análise das relações entre o IBOVESPA e os índices setoriais é de suma importância no que tange às decisões de investimentos dos agentes econômicos e financeiros, pois o enfoque setorial é primordial no que se refere à redução do risco de carteiras de ações, e considerando que o mercado financeiro (acionário) tem fortes reflexos sobre o lado real da economia.

Em face dos resultados obtidos, em trabalhos futuros pode-se buscar: i) trabalhar com um período de tempo maior, que cubra o período anterior e posterior à crise do subprime. Dessa forma, poderiam ser realizadas estimativas segmentadas por períodos, a fim de verificar se os resultados são mantidos em períodos distintos, como por exemplo: antes, durante e depois da crise do subprime; ii) adotar modelos que tratem a heteroscedasticidade condicional, comum em séries do mercado financeiro, e; iii) utilizar o método de wavelets (método de análise de ondaletas) e os métodos não paramétricos de causalidade de Granger, fazendo uma comparação com os resultados deste estudo.

\section{Referências}

BACA, S.; GARBE, B.; WEISS, R. The rise of sector effects in major equity markets. Financial Analysts Journal, v. 56, n. 5, p. 34-40, 2000. DOI: https://doi.org/ 10.2469/faj.v56.n5.2388 
BUENO, R. D. L. S. Econometria de séries temporais. 2. ed. São Paulo: Cengage Learning, 2011.

CAMPBELL, J. Y.; PERRON, P. Pitfalls and opportunities: what macroeconomists should know about unit roots. In: BLANCHARD, O. J.; FISCHER, S. (Ed.). NBER Macroeconomics annual. Cambridge, CA: The MIT Press, p. 141-201, 1991. DOI: https://doi.org/10.3386/t0100

CAMURI, P. A. Dívida pública, política fiscal e restrição externa no Brasil: 1994-2004. X Prêmio Tesouro Nacional, Belo Horizonte, 2005.

CASTRO, N. J.; BRANDÃO, R. A crise econômico-financeira e os impactos no setor elétrico brasileiro. Grupo de Estudos do Setor Elétrico - UFRJ. Disponível em: <http://www.nuca.ie.ufrj.br/gesel/>. Acesso em: 13 abr. 2018.

DICKEY, D. A.; FULLER, W. A. Likelihood ratio statistics for autoregressive time series with a unit root. Econometrica, v. 49, n. 4, p. 1057-1073, 1981. DOI: https://doi.org/10.2307/1912517

EWING, B. T. The response of the default risk premium to macroeconomic shocks. The Quarterly Review of Economics and Finance, v. 43, p. 261-272, 2003. DOI: https://doi.org/10.1016/S1062-9769(02)00147-3

ENGLE, R. F.; GRANGER, C. W. J. Cointegration and error correction: representation, estimation, and testing. Econometrica, v. 55, n. 2, p. 251-276, 1987. DOI: https://doi.org/10.2307/1913236

FAMA, E. The behaviour of stock prices. Journal of Business, v. 38, n. 1, p. 34-105, 1965. DOI: https://doi.org/10.1086/294743

FERREIRA, D. M.; MATTOS, L. B. O contágio da crise do subprime no mercado acionário brasileiro. In: $40^{\circ}$ ENCONTRO NACIONAL DE ECONOMIA, 2012, Porto de Galinhas. Anais eletrônicos.... Disponível em: http://www.anpec.org.br/ novosite/br/ encontro-2012. Acesso em: 15 de jul. 2016.

FUINHAS, J. A.; MARQUES, A. C.; NOGUEIRA, D. C. Integration of the indexes SP500, FTSE100, PSI20, HSI and IBOVESPA: a VAR approach. Paper, University Library of Munich, Germany. 2014. Disponível em:

<http://EconPapers.repec.org/RePEc:pra:mprapa:62092>. Acesso em: 2 dez. 2016. 
GENÇAY, R.; SELÇUK, F.; WHITCHER, B. An introduction to ondaletas and other filtering methods in finance and economics. New York: Academic Press, 2002. DOI: https://doi.org/10.1016/B978-012279670-8.50004-5

GRÔPPO, G. Causalidade das variáveis macroeconômicas sobre o IBOVESPA, Piracicaba, Brasil. 2004. 107 f. Dissertação (Mestrado em Economia Aplicada) Programa de Pós-Graduação em Economia Aplicada, Escola Superior de Agricultura Luiz de Queiroz, Universidade de São Paulo, Piracicaba, 2004.

HANSEN, H.; JUSELIUS, K. Cats in rats: cointegration analysis of time series. USA: Estima, 1995.

HERMANN, J.; MARTINS, N. M. Mercado de ações no Brasil: o perfil de risco da expansão de 2004-2011. Análise Econômica, v. 30, n. 58, p. 87-120, 2012.

JOHANSEN, S. Statistical analysis of cointegration vectors. Journal of Economic Dynamics and Control, v. 12, p. 231-254, 1988. DOI: https://doi.org/10.1016/01651889(88)90041-3

JUBERT, R.; MONTE, P.; PAIXÃO, M.; LIMA, W. Um estudo do padrão de volatilidade dos principais índices financeiros do BOVESPA: uma aplicação de modelos ARCH. Revista UnB Contábil, v. 11, n. 1-2, p. 221-239, 2008.

KOOP, G.; PESARAN, M. H.; POTTER, S. M. Impulse response analysis in nonlinear multivariate models. Journal of Econometrics, v. 74, p. 119-147, 1996. DOI: https://doi.org/10.1016/0304-4076(95)01753-4

KWIATKOWSKI, D.; PHILLIPS, P. C. B.; SCHMIDT, P.; SHIN, Y. Testing the null hypothesis of stationarity against the alternative of unit root. Journal of Econometrics, v. 54, n. 1, p. 159-178, 1992. DOI: https://doi.org/10.1016/0304-4076(92)90104-Y

LINTNER, J. Security prices, risk, and maximal gains from diversification. Journal of Finance, v. 20, n. 4, p. 587-615, 1965. DOI: https://doi.org/10.1111/j.15406261.1965.tb02930.x

LÜTKEPOH, H. New introduction to multiple time series analysis. New York, Springer, 2007.

MACEDO JUNIOR, J. S. Teoria do prospecto: uma investigação utilizando simulação de investimentos, Florianópolis, Brasil. 2003. 203 f. Tese (Doutorado em Engenharia da 
Produção) - Programa de Pós-Graduação em Engenharia da Produção, Universidade Federal de Santa Catarina, Florianópolis, 2003.

MACKINNON, J. C.; HAUG, A. A.; MICHELIS, L. Numerical distribution functions of likelihood ratio tests for cointegration. Journal of Applied Econometrics, v. 14, p. 563577, 1999. DOI: https://doi.org/10.1002/(SICI)1099-1255(199909/10) 14:5<563::AID-JAE530>3.0.C0;2-R

MANDELBROT, B. The variation of certain speculative prices. The Journal of Business, v. 36, p. 394-419, 1963. DOI: https://doi.org/10.1086/294632

MARKOWITZ, H. M. Portfolio Selection. Journal of Finance, v. 7, n. 1, p. 77-91, 1952. DOI: https://doi.org/10.1111/j.1540-6261.1952.tb01525.x

MARKOWITZ, H. M. Foundations of portfolio theory. Journal of Finance, v. 46, n. 2, p. 469- 477, 1991. DOI: https://doi.org/10.1111/j.1540-6261.1991.tb02669.x

MEDEIROS, LUIZ G. C. Análise quantitativa da volatilidade dos índices setoriais da BOVESPA através de modelos GARCH univariados. 2012. 77 f. Monografia (Graduação em Economia) - Faculdade de Ciências Econômicas, Universidade Federal do Rio Grande do Sul, Porto Alegre, 2012.

MOSSIN, J. Equilibrium in a capital asset market. Econometrica, v. 34, n. 4, p. 768-783, 1966. DOI: https://doi.org/10.2307/1910098

MÜLLER, H. H. Modern portfolio theory: some main results. ASTIN Bulletin: The Journal of the International Actuarial Association, v. 19, p. 9-27, 1989. DOI: https://doi.org/10.1017/S051503610000859X

NUNES, M. S.; COSTA Jr, N. C. A.; MEURERII, R. A relação entre o mercado de ações e as variáveis macroeconômicas: uma análise econométrica para o Brasil. Revista Brasileira de Economia, v. 59, n. 4, 2005. DOI: https://doi.org/10.1590/S003471402005000400004

OREIRO, J. L., PAULA, L. F., ONO, F. H.; SILVA, G. J. C. Determinantes macroeconômicos do spread bancário no Brasil: teoria e evidência recente. Economia Aplicada, v. 10, n. 4, p. 609-634, 2006. DOI: https://doi.org/10.1590/S1413-80502006000400007

PADMANABHAN, D.; SINHA, A. S.; VENKATARAMAN, A. V.; RAVI, A.; JOSHI, A. Comparative analysis of the stock markets of China, Russia, Brazil, South Africa and 
Argentina. Paper, University Library of Munich, Germany. 2015. Disponível em: $<$ https://mpra.ub.uni-muenchen.de/63440/1/MPRA_paper_63440.pdf>. Acesso em: 2 dez. 2015.

PESARAN, M. H.; SHIN, Y. Generalized impulse response analysis in linear multivariate models. Economics Letters, v. 58, p. 17-29, 1998. DOI:

https://doi.org/10.1016/S0165-1765(97)00214-0

PHILLIPS, P. C. B.; PERRON, P. Testing for unit roots in time series regression. Biometrika, v. 75, n. 3, p. 335-346, 1988. DOI: https://doi.org/10.1093/biomet/75.2.335

REBOREDO, J. C.; RIVERA-CASTRO, M. A.; UGOLINI, A. Wavelet-based test of comovement and causality between oil and renewable energy stock prices. Energy Economics, n. 61, p. 241-252, 2017. DOI:

https://doi.org/10.1016/j.eneco.2016.10.015

SHARPE, W. F. A simplified model for portfolio analysis. Management Science, v. 9, n. 2, p. 277-293, 1963. DOI: https://doi.org/10.1287/mnsc.9.2.277

SHARPE, W. F. Capital asset prices: a theory of market equilibrium under conditions of risk. Journal of Finance, v. 19, n. 3, p. 425-442, 1964. DOI: https://doi.org/10.1111/j.1540-6261.1964.tb02865.x

SILVA, J. X. S.; BARBEDO, C. H. S.; ARAÚJO, G. S. Há efeito manada em ações com alta liquidez do mercado brasileiro?. Brasília: Banco Central, 2015. (Texto para Discussão, 386). Disponível em: <https://www.bcb.gov.br/pec/wps/port/TD386.pdf>. Acesso em: 01 de dez. 2016. DOI: https://doi.org/10.15194/jofi_2015.v1.i2.16

SIMS, C. Macroeconomics and reality. Econometrica, v. 48, n. 1, p. 1-48, 1980. DOI: https://doi.org/10.2307/1912017

SIMS, C.; STOCK, J.; WATSON, M. Inference in linear time series models with some unit roots. Econometrica, v. 58, p. 113-144, 1990. DOI: https://doi.org/10.2307/2938337

TEIXEIRA, E. E. M. Relação entre as expectativas econômicas publicadas no relatório Focus e o retorno e volatilidade das principais ações negociadas no Brasil, Belo Horizonte, Brasil. 2011. 209 f. Tese (Doutorado em Administração) - Programa de Pós-Graduação e Pesquisas em Administração, Faculdade de Ciências Econômicas, Universidade Federal de Minas Gerais, Belo Horizonte, 2011. 
TOBIN, J. Liquidity preference as a behavior toward risk. Review of Economic Studies, v. 25, n. 2, p. 65-86, 1958. DOI: https://doi.org/10.2307/2296205

Recebido em 28.09.17

Aprovado em 16.05.18 